\title{
Possibilistic Evaluation of Extended Fuzzy Rules in the Presence of Uncertainty
}

\author{
Manolis Wallace ${ }^{1,2}$ and Stefanos Kollias ${ }^{1}$ \\ ${ }^{1}$ National Technical University of Athens, \\ 9, Iroon Polytechniou Str., 15780 Zographou, Athens, Greece \\ ${ }^{2}$ University of Indianapolis, Athens Campus, \\ 9, Ipitou Str., 10557 Syntagma, Athens, Greece
}

\begin{abstract}
Characterization fuzzy in term "fuzzy rule base" is currently referred to the ability to define rule antecedents using fuzzy numbers. On the other hand, when it comes to the knowledge described by the rules and to the information contained in rule antecedents, absolute accuracy is assumed. With the emergence of a vast variety of applications of rule based systems, where antecedents are not provided by sensors but rather by complicated processing modules, more efficient rules and rule evaluation structures are needed, that are able to describe knowledge in more intuitive manner and cope with uncertainty in the assumed input. In this paper we propose extended fuzzy rules that allow for optional antecedents and provide a methodology for the possibilistic evaluation of both conventional and extended fuzzy rules in the presence of uncertainty. The work has been successfully applied in a real life problem, for which conventional fuzzy rules and fuzzy rule evaluation were inadequate.
\end{abstract}

\section{INTRODUCTION}

Fuzzy rules and fuzzy rule base systems have been used extensively in the past in the design and implementation of expert systems, as they provide for a very intuitive way for expert users to formalize knowledge that can be then utilized to drive an automated intelligent system. The result is systems that can process complex inputs in a very short time and respond accordingly.

In cases where response time is critical, systems based on fuzzy rules are often considered as the obvious choice. Measurements received from sensors are mapped to high level linguistic variables, which are then used in order to provide for a quick approximation of the optimal response. The only required assumptions are that the optimal response is a continuous function of the considered inputs, the knowledge described by the fuzzy rules is correct and of course that the inputs are available.

Although these seem to be as reasonable and relaxed assumptions, cases exist in two out of these three assumptions cannot be met:

- If some inputs can be characterized as optional in certain cases, expert users typically have to either ignore that fact or totally disregard their existence, as the conventional fuzzy rule format does not allow for optional rule antecedents.

- When the inputs of an expert system are obtained as the output of another system, as is, for example, a video processing system, these inputs might be in some cases unavailable or uncertain.

In this paper, driven by a real life problem where the two abovementioned situations are observed, thus making conventional rule base systems inadequate, we extend fuzzy rule format and propose a novel fuzzy rule evaluation model that provides for the consideration of uncertain input. Specifically, in section II we discuss conventional rule format and present an extended fuzzy rule format that allows for optional rule antecedents. In section III we present our methodology for fuzzy rule evaluation under uncertainty; we explain how this evaluation model produces results that have a possibilistic nature, which also helps tackle the issue of concurrent activation of contradicting rules. Finally, in section IV we briefly present the real life problem that initially led us to this work and further served as the experimental test bed for the presented methodology, and in section $\mathrm{V}$ we present our concluding remarks.

\section{KNOWLEDGE REPRESENTATION.}

Expert systems are knowledge based systems that try to emulate the consulting or decision making operation of a human expert. An essential issue in their design and operation is the representation of the knowledge they contain in an intuitive and human friendly format, so that human experts can easily describe the knowledge or examine and validate it after it has been automatically generated or refined by the system through supervised or unsupervised training.

Fuzzy rule base systems are expert systems that typically contain knowledge in the form of rules such as the following:

$$
\text { IF } x_{1}, x_{2}, \ldots, x_{n} \text { THEN } y
$$

where $y$ is the consequent of the rule and $x_{1}, x_{2}, \ldots, x_{n}$ are the antecedents of the rule. The antecedents typically follow the form: 


$$
x_{i}: f_{i} \text { IS } X_{i}
$$

where $f_{i}$ is a measurable feature and $X_{i}$ is a fuzzy number quantifying the measurement. Similarly, the consequent follows the form:

$$
y: o \text { IS } Y
$$

where $O$ is the output case described by the rule and $Y$ is a fuzzy number quantifying the output. For example, a rule that could be contained in a fuzzy rule base system is the following:

IF temprature IS high_temp

AND humidity IS high_hum

THEN it_feels_hot IS true

In this case, the count $n$ of rule antecedents is $n=2$, the measurable feature $f_{1}$ of the first antecedent is the temperature, the fuzzy number $X_{1}$ quantifying the temperature is 'high_temp', the output case $o$ described by the rule is 'it_feels_hot', the fuzzy number $Y$ quantifying the output is 'true' and so on. This rule is characterized in the literature as a fuzzy rule due to the fact that the quantification of the measurable features $f_{i}$ by fuzzy numbers $X_{i}$ is not crisp, i.e. it contains degrees. For example, as can be seen from Figure 1, a temperature measurement between twenty seven and thirty degrees will be quantified as high_temp to a degree other than zero or one.

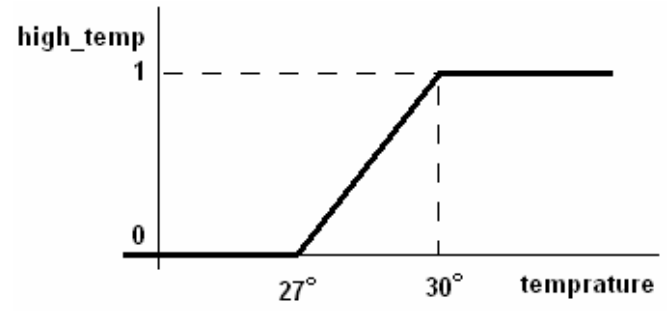

Figure 1. high_temp fuzzy number

The knowledge itself, on the other hand, as described by the rule, is not at all fuzzy; the rule describes that high temperature and high humidity result in a hot feeling, but cannot express, for example, that high temperature alone could have the same result, and thus that high humidity is an optional component with the sole result of intensifying the feeling. A first step towards tackling this problem is presented in [5], where degrees of importance are assigned to different rule antecedents. The solution, though, is not complete, as in order for complete absence of the optional antecedent not to make impossible the activation of the rule with degree 1 , the importance of the antecedent needs to be set to 0 ; by doing this the antecedent is affectively removed from the rule and is not considered even when it is present.

Term "weighted fuzzy rule" is also met in the literature, but it does not refer to weighting of rule antecedents, which could be seen as an approach to tackling this problem. It rather refers to assigning degrees of confidence to the rule as a whole in the case of a rule that contains doubted knowledge, as is often the case with rules that have been automatically generated through some unsupervised data mining procedure.

Of course, conventional fuzzy rules could also handle such a constraint with a number of tricks, all of which have serious limitations and drawbacks:

Trick 1: The optional component can be removed from the rule. In this case the rule successfully describes that a measurement of high temperature alone is enough to determine a hot feeling. On the other hand, in the cases that a measurement of high humidity is also available, this rule will ignore this important information bit, similarly to using the rule structure of [5] and assigning importance 0 to the antecedent.

Trick 2: Two independent rules can be included in the rule base: one containing only the temperature as an antecedent and one containing both the temperature and the humidity as antecedents and providing a higher output estimation. This has the drawbacks that

- it results in a more complex rule based system,

- rules are trained independently and possibly in different ways when incorporated in a learning neurofuzzy structure,

- depending on the training methodology followed, the rules could be competitive to each other despite the fact that they describe quite similar situations and

- this representation makes it more complicated and less intuitive for an expert to create or examine and verify the rules.

The case considered herein, i.e. the case of optional but not negligible antecedents, can be described successfully and in a very intuitive manner using rules of the form:

IF temprature IS high_temp

THEN it_feels_hot IS true

MORE SO IF humidity IS high_hum

Thus, in this work we extend the conventional fuzzy rule format of (1.1) as follows:

$$
\begin{aligned}
& \text { IF } x_{1}, x_{2}, \ldots, x_{n} \text { THEN } y \text { MORE SO IF } x_{n+1}, x_{n+2}, \ldots, x_{m}(1.6) \\
& \text { where } x_{n+1}, x_{n+2}, \ldots, x_{m} \text { correspond to the optional }
\end{aligned}
$$
components of the rule and, similarly to $x_{1}, x_{2}, \ldots, x_{n}$, are of the form:

$$
x_{i}: f_{i} \text { IS } X_{i}
$$

\section{Possibilistic Rule EVAluAtion}

In the temperature example provided in the previous section, measurement $f_{1}$ of the temperature is assumed to be available with absolute precision, so that the membership function of Figure 1 can be applied. Although this is a reasonable assumption for sensor driven fuzzy systems, systems that are driven by complex, imprecise and uncertain output cannot be assumed to fulfill it.

In many real life problems, such as facial expression estimation that we present in section IV, a number of delicate issues has to be considered, such as: 
- the case of antecedent values that cannot be estimated,

- the case of antecedents estimated with a low degree of confidence and

- the activation of contradicting rules.

A conventional approach to the evaluation of fuzzy rules of the form described in equation (1.1) is as follows:

$$
y=t\left(x_{1}, x_{2}, \ldots, x_{n}\right)
$$

where $t$ is a fuzzy $t$-norm, such as the minimum

$$
t\left(x_{1}, x_{2}, \ldots, x_{n}\right)=\min \left(x_{1}, x_{2}, \ldots, x_{n}\right)
$$

the algebraic product

$$
t\left(x_{1}, x_{2}, \ldots, x_{n}\right)=x_{1} \cdot x_{2} \cdot \ldots \cdot x_{n}
$$

the bounded sum

$$
t\left(x_{1}, x_{2}, \ldots, x_{n}\right)=x_{1}+x_{2}+\ldots+x_{n}+1-n
$$

and so on. Another well known approach in rule evaluation is described in [4] and utilizes a weighted sum instead of a $t$ norm in order to combine information from different rule antecedents:

$$
y=w_{1} x_{1}+w_{2} x_{2}+\ldots+w_{n} x_{n}
$$

Both approaches are well studied and established in the field of fuzzy automatic control. Still, they are not adequate for the case of problems such as facial expression estimation: their main disadvantage is that they assume that all antecedents are known, i.e. that all features $f_{1}$ are measured successfully and precisely. In the case of facial expression estimation, on the other hand, feature points may well be estimated with a very low confidence, or not estimated at all, due to low video quality, speech interference, occlusion, noise and so on; similar problems can of course be met in various other complex rule based systems. Thus, a more flexible rule evaluation scheme that is able to incorporate this uncertainty is required for such cases.

Moreover, the second one of the conventional approaches, due to the summation form, has the disadvantage of possibly providing a highly activated output even in the case that an important antecedent is known to be missing; obviously it is not suitable for the case of problems of the type examined in this paper; for example, the non activation of a facial animation parameter automatically implies that the expression profiles that require it are not activated either. Therefore, the ideal rule evaluation scheme should in fact be a generalization of the $t$-norm based conventional approach of equation (1.8).

\section{A. Possibilistic Evaluation of Required Antecedents}

In the $t$-norm operation described in equation (1.8), antecedents with lower values affect most the resulting value of $y$, while antecedents with values close to one have trivial and negligible affect on the value of $y$. Having that in mind, we can demand that only antecedents that are known with a high confidence will be allowed to have low values in that operation. More formally, we demand that the degree $k(x)$ to which antecedent $x$ is considered is low when the confidence $x^{c}$ with which the value of $x$ is known is high and the value of $X$ is low; depending on the type of application, the degree of confidence $x^{c}$ may be either provided directly by the sensor or, most probably, estimated via some step of input validation against known criteria [8]. This can be expressed as:

$$
c(k(x))=x^{c} \cap c(x)
$$

where $c$ is a fuzzy complement. Applying de Morgan's law we have that the degree to which antecedent $x$ is considered is:

$$
k(x)=c\left(x^{c}\right) \cup x
$$

It is easy to see that equation (1.14) satisfies the desired marginal conditions:

- when $x^{c} \rightarrow 1$, then $c\left(x^{c}\right) \rightarrow 0$ and $k(x) \rightarrow x$, i.e. the antecedent is considered normally, while

- when $x^{c} \rightarrow 0$, then $c\left(x^{c}\right) \rightarrow 1$ and $k(x) \rightarrow 1$, i.e. the antecedent is not allowed to affect the overall evaluation of the rule; the formula that provides the overall evaluation assumed in this discussion is the one followed by the conventional approach, with the exception that antecedents participate with their considered values:

$$
y=t\left(k\left(x_{1}\right), k\left(x_{2}\right), \ldots, k\left(x_{n}\right)\right)
$$

It is easy to see that in the case that all antecedents are known with a confidence of 1 the rule will be evaluated in the same way as in the conventional methodology. When, on the other hand, all antecedents are known with a confidence of zero, i.e. when no information is available, the rule will be evaluated with a degree of 1 . Thus, the activation level of a rule with this approach can be interpreted in a possibilistic manner, i.e. it can be interpreted as the degree to which the corresponding output is possible, according to the available information; in the literature, this possibilistic degree is referred to as plausibility [3].

As far as the confidence in the calculated output is concerned, in the conventional approach we either always assume total confidence in the output, which originates from the assumption that all inputs are precisely known. In the extended approach followed herein, where we accept that one or more of the required rule antecedents may be unknown or known with a confidence other than zero, it does not make sense to always assume total confidence in the computed activation level. Quite the contrary, the calculated activation level is only complete in information when associated with a corresponding degree of confidence.

The confidence is determined by the confidence values of the utilized inputs, i.e. by the confidence values of the rule antecedents, as follows:

$$
y^{c}=\frac{x_{1}^{c}+x_{2}^{c}+\ldots+x_{n}^{c}}{n}
$$

The definition of $y^{c}$ in this manner has the desired effect that $y^{c}=0$ is equivalent to the complete lack of information; this property is essential in possibilistic reasoning [3]. 


\section{B. Consideration of Optional Antecedents}

The abovementioned approach is sufficient for the evaluation not only of rules of the conventional form described in equation (1.1), but also for the evaluation of the first part of the extended rules described in equation (1.5). As far as the evaluation of the contribution of the remaining of the rule is concerned, it should not be allowed to effect the overall confidence, which should only be affected by the confidence in the mandatory antecedents of the rule. On the other hand, when it comes to the level of activation of the rule, i.e. to the plausibility of the corresponding expression profile, the detection of an optional antecedent, to any degree and with any confidence, should not be allowed to lessen the activation level calculated at the absence of that antecedent. Thus, optional antecedents operate as weak modifiers [3], only augmenting the calculated activation level. Of course, an optional antecedent should only be allowed to have a high affect on the calculated activation level if the antecedent has a high value and is known with a high confidence. More formally, the degree $l(x)$ to which the optional antecedent $x$ should be considered can be estimated as

$$
l(x)=t\left(x, x^{c}\right)
$$

The detection of optional antecedent $x$ should augment the activation level calculated using the mandatory antecedents as follows:

$$
y^{\prime}=H(y, l(x))
$$

where $H$ is a parameterized weak modifier [3]; modifier $H$ is applied to the first argument $y$, and parameter $l(x)$ is used in such a manner that when it assumes high values the affect of the modifier is maximized. In this work we have considered the following parameterized weak modifier for the evaluation of the developed methodology:

$$
H(a, b)=a^{\frac{1}{1+b}}
$$

In order to consider multiple optional components, the corresponding weak modifiers are applied sequentially to the calculated activation level.

Summarizing, the activation level $y$ and confidence level

$y^{c}$ for the evaluation of an extended rule, i.e. for a rule following the form of equation (1.6), are as follows:

$$
\begin{gathered}
y=y_{m} \\
y_{i}=\left(y_{i-1}\right)^{\frac{1}{1+l\left(x_{i}\right)}}, i=m, m-1, . ., n+1 \\
l\left(x_{i}\right)=t\left(x_{i}, x_{i}^{c}\right) \\
y_{n}=t\left(k\left(x_{1}\right), k\left(x_{2}\right), \ldots, k\left(x_{n}\right)\right) \\
k(x)=c\left(x^{c}\right) \cup x \\
y^{c}=t\left(x_{1}^{c}, x_{2}^{c}, \ldots, x_{n}^{c}\right)
\end{gathered}
$$

\section{On the possibilistic nature of the evaluation}

When using the conventional rule evaluation methodology of (1.8), it is not rare to obtain crisp results by selecting the rule that was activated to the highest degree and ignoring the rest. This means that the conventional interpretation of the evaluation of the fuzzy rule has a probabilistic nature. This can be seen more clearly for the fact that:

- the rule with the highest activation is considered most probable than the others and

- cases where two rules are activated to almost equal degrees are not considered "clear" as both rules are considered almost equally probable.

On the other hand, it is worth noting that the output of a fuzzy rule base system, although has a probabilistic flavor, cannot be used as a formal probability measure as it does not meet the axiomatic probability definition. For example, the sum of the activation of contradicting rules is not guaranteed to be less than unity. Similarly, we cannot claim that the output of the proposed possibilistic rule evaluation meets all the formal requirements to be named possibilistic. Still, it is clear that it has a possibilistic nature.

Thus, the activation level of the rule has a possibilistic interpretation and corresponds to the plausibility of the rule. In order, of course, to have a complete possibilistic representation of the rule evaluation, together with the plausibility of the rule we need to estimate the corresponding belief, i.e. the degree to which available evidence suggests that the output case described by the rule is verified by the available input.

The belief measure should be high when sufficient information is available during the evaluation of the rule, and that information verifies the output case described by the rule. The amount of information that is available during the evaluation of the rule is provided by the calculated confidence value, while the degree to which this information verifies the output case described by the rule is provided by the activation level. Thus, the complete possibilistic representation of the calculated output is provided as:

$$
\begin{gathered}
\mathrm{Bel}=t\left(y, y^{c}\right) \\
P l=y^{c}
\end{gathered}
$$

The case of activation of multiple and contradicting rules of the rule base is not an issue for this approach. In that case, it is expected that confidence values will be low, which can be interpreted as the case in which, due to poor input, more than one possible output cases cannot be ruled out. Still, the belief that they a indeed be activated, as reported by equation (1.26), will be low.

\section{EXPERIMENTAL TEST BED}

The goal of IST project ERMIS is the development of a prototype system for human computer interaction than can interpret its users' attitude or emotional state, e.g., interest, boredom, anger, etc. in terms of their speech and their facial gestures and expressions [9]. In this framework, a software prototype of the expert system has been developed that is able to automatically categorize facial expressions observed on real faces. The proposed extended rule model and possibilistic rule evaluation have been incorporated into this prototype. 
In order to utilize extended fuzzy rules, or even the conventional fuzzy rule format, in describing the knowledge about human expressions that is needed by the system in estimating the expression of the user starting from raw media input such as static images or video sequences in a Man Machine Interaction (MMI) context, we have first described the six archetypal expressions (joy, sadness, anger, fear, disgust, surprise) in a symbolic manner, using easily and robustly estimated tokens; Facial Animation Parameter (FAP) representations, as explained in [8], make good candidates for describing quantitative facial motion features. The use of these parameters serves several purposes such as compatibility with the MPEG-4 standard and increase of the range of the described expressions; archetypal expressions occur rather infrequently and in most cases expressions are expressed through variation of a few discrete facial features related with particular FAPs.

Table 1. FAP IDs and names

\begin{tabular}{|l|l|l|l|}
\hline FAP & Full name of FAP & FAP & Full name of FAP \\
\hline $\mathrm{F}_{3}$ & open_jaw & $\mathrm{F}_{4}$ & lower_t_midlip \\
\hline $\mathrm{F}_{5}$ & raise_b_midlip & $\mathrm{F}_{6}$ & stretch_l_cornerlip \\
\hline $\mathrm{F}_{7}$ & stretch_r_cornerlip & $\mathrm{F}_{8}$ & lower_t_lip_lm \\
\hline $\mathrm{F}_{9}$ & lower_t_lip_rm & $\mathrm{F}_{10}$ & raise_b_lip_lm \\
\hline $\mathrm{F}_{11}$ & raise_b_lip_rm & $\mathrm{F}_{12}$ & raise_l_cornerlip \\
\hline $\mathrm{F}_{13}$ & raise_r_cornerlip & $\mathrm{F}_{16}$ & push_b_lip \\
\hline $\mathrm{F}_{18}$ & Depress_chin & $\mathrm{F}_{19}$ & close_t_l_eyelid \\
\hline $\mathrm{F}_{20}$ & close_t_r_eyelid & $\mathrm{F}_{21}$ & close_b_l_eyelid \\
\hline $\mathrm{F}_{22}$ & close_b_r_eyelid & $\mathrm{F}_{31}$ & raise_l_i_eyebrow \\
\hline $\mathrm{F}_{32}$ & raise_r_i_eyebrow & $\mathrm{F}_{33}$ & raise_l_m_eyebrow \\
\hline $\mathrm{F}_{34}$ & raise_r_m_eyebrow & $\mathrm{F}_{35}$ & raise_l_o_eyebrow \\
\hline $\mathrm{F}_{36}$ & raise_r_o_eyebrow & $\mathrm{F}_{37}$ & squeeze_l_eyebrow \\
\hline $\mathrm{F}_{38}$ & Squeeze_r_eyebrow & $\mathrm{F}_{41}$ & lift_l_cheek \\
\hline $\mathrm{F}_{42}$ & lift_r_cheek & $\mathrm{F}_{53}$ & stretch_l_cornerlip_o \\
\hline $\mathrm{F}_{54}$ & Stretch_r_cornerlip_0 & $\mathrm{F}_{55}$ & lower_t_lip_lm_o \\
\hline $\mathrm{F}_{56}$ & lower_t_lip_rm_0 & $\mathrm{F}_{57}$ & raise_b_lip_lm_o \\
\hline $\mathrm{F}_{58}$ & raise_b_lip_rm_o & $\mathrm{F}_{59}$ & raise_l_cornerlip_o \\
\hline $\mathrm{F}_{60}$ & raise_r_cornerlip_0 & & \\
\hline
\end{tabular}

Based on elements from psychological studies [1][2][6][7], we have described the six archetypal expressions using MPEG-4 FAPs; the list of utilized FAPs for each one of the archetypal expressions forms the expression vocabulary and is illustrated in Table 2 while FAP names corresponding to FAP IDs of Table 2 are reported in Table 1. FAP names have been chosen to be descriptive, in order to facilitate manual specification and examination of the rule base; for example FAP $F_{13}$ with name "raise_r_cornerlip" corresponds to the raising of the right corner of the lip. In general, these expressions can be uniformly recognized across cultures and are therefore invaluable in trying to analyze the users' emotional state.

As a general rule, one can define six general expression categories, each characterized by an archetypal expression; within each of these categories, intermediate expressions are described by different emotional intensities, as well as minor variations in expression details. From the processing point of view, expressions belonging to the same category can be represented using the same FAPs as in the case of the archetypal expression, but using different intensities. This leads to the generation of various expression profiles, each one describing different levels of activation of the expression vocabulary FAPs; each expression profile is represented using a distinct extended fuzzy rule.

Table 2. FAPs vocabulary for archetypal expression description

\begin{tabular}{|l|l|}
\hline Joy & $\mathrm{F}_{3}, \mathrm{~F}_{4}, \mathrm{~F}_{5}, \mathrm{~F}_{6}, \mathrm{~F}_{7}, \mathrm{~F}_{12}, \mathrm{~F}_{13}, \mathrm{~F}_{19}, \mathrm{~F}_{20}, \mathrm{~F}_{21}, \mathrm{~F}_{22}, \mathrm{~F}_{33}, \mathrm{~F}_{34}$, \\
\hline Sadness & $\mathrm{F}_{41}, \mathrm{~F}_{42}, \mathrm{~F}_{53}, \mathrm{~F}_{54}, \mathrm{~F}_{20}, \mathrm{~F}_{21}, \mathrm{~F}_{22}, \mathrm{~F}_{31}, \mathrm{~F}_{32}, \mathrm{~F}_{33}, \mathrm{~F}_{34}, \mathrm{~F}_{35}, \mathrm{~F}_{36}$ \\
\hline Anger & $\mathrm{F}_{4}, \mathrm{~F}_{5}, \mathrm{~F}_{16}, \mathrm{~F}_{18}, \mathrm{~F}_{19}, \mathrm{~F}_{20}, \mathrm{~F}_{21}, \mathrm{~F}_{22}, \mathrm{~F}_{31}, \mathrm{~F}_{32}, \mathrm{~F}_{33}, \mathrm{~F}_{34}$, \\
& $\mathrm{F}_{35}, \mathrm{~F}_{36}, \mathrm{~F}_{37}, \mathrm{~F}_{38}$ \\
\hline Fear & $\mathrm{F}_{3}, \mathrm{~F}_{4}, \mathrm{~F}_{5}, \mathrm{~F}_{8}, \mathrm{~F}_{9}, \mathrm{~F}_{10}, \mathrm{~F}_{11}, \mathrm{~F}_{19}, \mathrm{~F}_{20}, \mathrm{~F}_{21}, \mathrm{~F}_{22}, \mathrm{~F}_{31}, \mathrm{~F}_{32}$, \\
& $\mathrm{F}_{33}, \mathrm{~F}_{34}, \mathrm{~F}_{35}, \mathrm{~F}_{36}, \mathrm{~F}_{37}, \mathrm{~F}_{38}$ \\
\hline Disgust & $\mathrm{F}_{3}, \mathrm{~F}_{4}, \mathrm{~F}_{5}, \mathrm{~F}_{8}, \mathrm{~F}_{9}, \mathrm{~F}_{10}, \mathrm{~F}_{11}, \mathrm{~F}_{19}, \mathrm{~F}_{20}, \mathrm{~F}_{21}, \mathrm{~F}_{22}, \mathrm{~F}_{33}, \mathrm{~F}_{34}$, \\
& $\mathrm{F}_{55}, \mathrm{~F}_{56}, \mathrm{~F}_{57}, \mathrm{~F}_{58}, \mathrm{~F}_{59}, \mathrm{~F}_{60}$ \\
\hline Surprise & $\mathrm{F}_{3}, \mathrm{~F}_{5}, \mathrm{~F}_{6}, \mathrm{~F}_{7}, \mathrm{~F}_{10}, \mathrm{~F}_{11}, \mathrm{~F}_{19}, \mathrm{~F}_{20}, \mathrm{~F}_{21}, \mathrm{~F}_{22}, \mathrm{~F}_{31}, \mathrm{~F}_{32}$, \\
& $\mathrm{F}_{33}, \mathrm{~F}_{34}, \mathrm{~F}_{35}, \mathrm{~F}_{36}, \mathrm{~F}_{37}, \mathrm{~F}_{38}, \mathrm{~F}_{53}, \mathrm{~F}_{54}$ \\
\hline
\end{tabular}

On the other hand, the process of image processing of facial images leads to the measurement of some or all of these FAPs, in different scales and ranges. Thus, each FAP is quantified using different fuzzy numbers, in order to acquire the antecedents required for the evaluation of the fuzzy rules; each expression profile is represented using a distinct fuzzy rule. Each FAP may theoretically be quantified using a different fuzzy number for each rule in which it participates; in practice only a few fuzzy numbers are used for each FAP and are shared among rules, which greatly simplifies the generation and inspection of the rule base by human experts. For example, FAP $\mathrm{F}_{42}$ (lift_r_cheek) is quantified using only fuzzy numbers "raised" and "not_raised", while FAP $F_{3}$ (open_jaw) is quantified using fuzzy numbers "high", "medium", "low" and "neutral".

Definition of the ranges of the FAPs is based on anthropometric data and specification of the optional antecedents of rules is based on the aforementioned psychological studies. Thus, for example, FAPs $\mathrm{F}_{35}$ and $\mathrm{F}_{36}$ are optional in most extended rules corresponding to profiles of the archetypal expression of fear.

As can be seen from the sample frame presented in Figure 2, feature points are not always identified successfully. In Figure 2, for example, all FAPs related to the mouth will be misestimated. Problems like this made the original version of the prototype, which was using the conventional rule evaluation methodology unable to handle the case and often provided random or obviously erroneous responses, insufficient. The updated version, equipped with the extended fuzzy rule structure and possibilistic fuzzy rule evaluation described in this work, never fails to produce meaningful output. Even in cases that the detected features are insufficient for any assumption on the use expression, the model successfully describes this lack of input through the high plausibility and low belief measures; in this way consequent processes that rely on the output of the fuzzy expert system are 
advised to disregards the evaluation of the specific frames and the error is not further propagated.

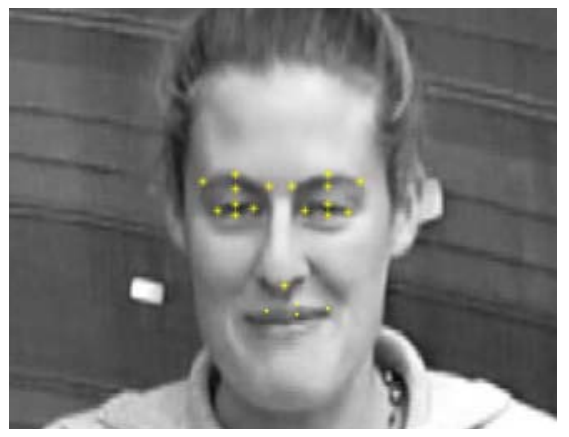

Figure 2. A sample frame

\section{CONCLUSiOnS}

We have started this paper by proposing an extended format for fuzzy rules that allows for optional rule antecedents. This format provides for a more simple and intuitive handling of optional antecedents, than the possible solutions when using the conventional fuzzy rule format.

Continuing, we have examined the case when one or more of the antecedents of a fuzzy rule are either unknown or known with some confidence other than 1 . We have proposed a flexible rule evaluation model that considers the confidence with which measured inputs are known.

This model, together with the estimation of the degree of activation of the rule, also provides a measure of the confidence in the considered input; the two measures combined constitute a possibilistic rule evaluation, as both belief and plausibility are provided.

The proposed fuzzy rule formal and flexible rule evaluation methodology have been used in the development of a fuzzy rule base system for the automatic analysis of raw video and audio input for the estimation of facial expressions. Although traditional fuzzy rules were not sufficient for this problem, where rule antecedents are often not available or computed with low certainty, the proposed approach has been proven to be successful.
Of course, we do not believe that the considered example is the only possible field of application for the proposed possibilistic fuzzy rule evaluation methodology. Quite the contrary, we believe that it could be considered for all fuzzy rule base systems that receive their input from some imperfect processing system. As future work, we intend to investigate the application of the proposed methodology in the design and implementation of other systems that are driven by an unsupervised video processing methodology, as is for example the matching of abstract object models in a knowledge base to features automatically extracted from low quality images or video sequences.

\section{ACKNOWLEDGEMENT}

This work was supported in part by the Greek ministry of education under the HERACLETUS Grant for basic research.

\section{References}

[1] Ekman, P., Facial expression and Emotion. Am. Psychologist, Vol. 48, 1993.

[2] Faigin, G., The Artist's Complete Guide to Facial Expressions, WatsonGuptill, New York, 1990.

[3] Klir G., Yuan B., Fuzzy Sets and Fuzzy Logic, Theory and Applications, New Jersey, Prentice Hall, 1995.

[4] Lee M.A., Takagi H., Integrating design stages of fuzzy systems using genetic algorithms, proceedings of IEEE International conference on fuzzy systems, 1993.

[5] Chen S.M., A weighted fuzzy reasoning algorithm for medical diagnosis, Decision Support Systems 11, pp. 37-43, 1994.

[6] Parke, F., Waters, K., Computer Facial Animation, A K Peters, 1996.

[7] Plutchik R., Emotion: A psychoevolutionary synthesis, Harper and Row, NY, USA, 1980.

[8] Ioannou S., Wallace M., Dealing with Feature Uncertainty in Facial Expression Recognition, IEEE International Conference on Fuzzy Systems, Reno, Nevada, 2005.

[9] IST Project: Emotionally Rich Man-Machine Interaction Systems (ERMIS), 2001-2003. http://www.image.ntua.gr/ermis/ 